Meta

Journal des tradlucteurs

Translators' Journal

\title{
Désignations de quelques postes de la hiérarchie hôtelière
}

\section{Service de linguistique du Canadien Pacifique}

Volume 16, numéro 3, septembre 1971

URI : https://id.erudit.org/iderudit/004077ar

DOI : https://doi.org/10.7202/004077ar

Aller au sommaire du numéro

Éditeur(s)

Les Presses de l'Université de Montréal

ISSN

0026-0452 (imprimé)

1492-1421 (numérique)

Découvrir la revue

Citer cet article

Service de linguistique du Canadien Pacifique (1971). Désignations de quelques postes de la hiérarchie hôtelière. Meta, 16(3), 172-174.

https://doi.org/10.7202/004077ar d'utilisation que vous pouvez consulter en ligne. 


\section{DÉSIGNATIONS DE QUELQUES POSTES \\ DE LA HIÉRARCHIE HÔ'TELIËRE}

Bellman - Chasseur - Bellman, synonyme de bellboy et de bellhop, désigne le garçon qui, dans un hôtel, porte les bagages jusqu'à la chambre du client et fait les courses. Le porteur (porter) n'assure la manutention des bagages que de l'entrée au hall de l'hôtel et du hall à la sortie.

Breakfast Cook - Cuisinier du matin - Employé d'hôtel préposé à la préparation du petit déjeuner.

Busboy — Débarrasseur/Aide-serveur — Garçon de restaurant qui débarrasse les tables après les repas (E. Deak, Grand dictionnaire d'américanismes).

3. Barthe, op. cit.

4. Barthe, op cit. 
En réalité, le rôle de busboy ne se limite pas à desservir la table : c'est également lui qui dresse et prépare les tables à l'avance, qui va à la cuisine chercher les plats et les apporte à la desserte où il aide le serveur à parfaire leur décoration. En un mot, il exécute toutes les menues besognes dont le serveur ne pourrait se charger sans nuire à la qualité du service à table. C'est précisément ce que renferme l'étymologie du mot busboy, apocope de omnibus boy, garçon à tout faire. L'appellation aide-serveur ${ }^{1}$ rend un compte exact de la fonction.

Coffeeman - Cafetier - Celui qui prépare le café. Naguère remplie par le cuisinier, cette tâche est, à notre époque de spécialisation, confiée à un spécialiste, ce qui justifierait l'emploi d'un néologisme de sens, le cafetier.

Collar Finisher - Glaceuse de cols - Employée chargée d'assurer le glaçage au fer à repasser.

Fry Cook - Friteur - Dans les grands hôtels ou restaurants, cuisinier spécialement chargé des fritures (Larousse encyclopédique). Ne pas confondre avec le friturier qui, lui, fait et vend de la friture - keeper of a fried fish or chip-potato shop (Harrap).

Gardemanger - Garde-manger - Selon le Webster, employé de grand restaurant ou hôtel chargé de la préparation des plats de viandes froides. Les dictionnaires français définissent le garde-manger comme étant un local ou un meuble. Par contre, dans l'ouvrage de Marcel Gautier (l'Industrie hôtelière, "Que sais-je?», p. 65), le garde-manger est au nombre des chefs de cuisine et chefs de partie. Marcel Gautier a-t-il voulu ainsi faire revivre une acception remontant au XIV siècle et relevée par Littré : «Au commun, trois queux [cuisiniers], dont il y aura toujours les deux à cour, et sera l'un gardemangier. »

Housekeeper - Gouvernante - Professionnelle ayant la haute main sur le personnel d'entretien et de service dans un hôtel ${ }^{2}$. Dans le même ouvrage, l'appellation «ménagère » qualifie la personne procédant chez un particulier aux divers travaux de propreté des locaux. Il y a donc lieu de distinguer.

Houseman - Préposé - Employé à qui l'on confie des travaux courants de nettoyage et de manutention dans un hôtel. Préposé, défini par Robert de la façon suivante : agent d'exécution subalterne, nous semble constituer la meilleure traduction de houseman.

Linenman - Garçon de lingerie - Employé chargé des courses au service de la lingère.

Maid - Femme de chambre - Personne qui nettoie et entretient les chambres des clients dans des hôtels ou autres établissements analogues. Rappelons que «bonne» et «servante» désignent des employées qui vivent chez leur maître.

Potwasher - Laveur de marmites - Il ne faudrait pas croire que potwasher est synonyme de dishwasher, car dans les grands hôtels les plongeurs ont été remplacés par des appareils. L'appellation potwasher désigne la personne chargée exclusivement du récurage des marmites. Le vieux mot marmiton est exactement

1. Voir aussi : Gérard Dagenais, Dictionnaire des difficultés de la langue française au Canada, Québec, Pedagogia, 1967, p. 333.

2. Nomenclature des métiers et des activités individuelles, Institut national de la statistique et des études économiques, Imprimerie nationale, Paris, 1962, p. 243. 
celui qui désigne cette fonction, mais il a acquis dans le langage moderne un sens péjoratif qui ferait sourire. Pourtant il serait peut-être bon de tenter une restitution.

Room Service Telephone Operator - Téléphoniste du service à l'étage Personne chargée de répondre aux appels téléphoniques provenant des chambres et consistant surtout en commandes de consommations et de repas.

Service Bartender - Garçon de bar - Personne assurant, dans un bar d'hôtel, le service des boissons apéritives, digestives et rafraîchissantes ${ }^{3}$.

Stillroom Girl - Officière - Stillroom désigne l'office, pièce attenante à la cuisine où se prépare le service de la table, d'où l'appellation «officière » qui se retrouve d'ailleurs à la page 243 de la Nomenclature des métiers déjà citée. 